\title{
Evaluation of the Performance of Direct Susceptibility Test by VITEK-2 from Positively Flagged Blood Culture Broth for Gram-Negative Bacilli
}

\author{
Kavipriya D ${ }^{1}$ Suman Susan Prakash ${ }^{1} \quad$ Sarumathi Dhandapani ${ }^{1} \quad$ Deepashree Rajshekar ${ }^{1}$ \\ Apurba Sankar Sastry ${ }^{1}$ \\ ${ }^{1}$ Department of Microbiology, Jawaharlal Institute of Postgraduate \\ Address for correspondence Dr. Apurba Sankar Sastry, MD, DNB, \\ Medical Education and Research, Puducherry, India \\ MNAMS, Dhanvantri Nagar, JIPMER Campus, Jawaharlal Institute \\ of Postgraduate Medical Education and Research, Puducherry - \\ 605006, India (e-mail: drapurbasastry@gmail.com).
}

J Lab Physicians 2021;13:374-379.

\author{
Abstract \\ Keywords \\ - antimicrobial suscep- \\ tibility testing \\ - bloodstream \\ infections \\ - categorical agreement \\ - essential agreement \\ - Enterobacteriaceae \\ - nonfermenters \\ - Vitek-2
}

Background Timely initiation of antimicrobial therapy in patients with blood stream infection is absolutely necessary to reduce mortality and morbidity. Most clinical microbiology laboratories use conventional methods for identification and antimicrobial susceptibility testing (AST) that involve biochemical methods for identification followed by AST by disk diffusion. The aim of the current study is to assess the various errors associated with direct susceptibility testing done from blood culture broth using automated AST system-Vitek-2 compact compared with the reference method of AST done from bacterial colonies.

Materials and Methods The study was conducted in a tertiary care public sector 2,200-bedded hospital in South India for a period of 6 months. The study involved positively flagged blood culture bottles that yielded single morphotype of Gram-negative organism by Gram stain. A total of 120 bacterial isolates were collected that consisted of consecutively obtained first 60 isolates of Enterobacteriaceae family (30 Escherichia coli and 30 Klebsiella pneumoniae) and consecutively obtained first 60 nonfermenters (30 Pseudomonas aeruginosa and 30 Acinetobacter baumannii). Vitek-2 AST was done from these 120 blood culture broth, following the protocol by Biomerieux, and results were obtained. Then, Vitek-2 was done from colonies (reference method) using appropriate panel for Enterobacteriaceae and nonfermenters, and results were obtained. Both the results were compared.

Results Nonfermenters showed a better categorical agreement of $97.6 \%$, as compared to Enterobacteriaceae, which showed 97\%. Among Enterobacteriaceae, both $E$. coli and K. pneumoniae showed categorical agreement of $97 \%$ each.

Conclusion The procedure of AST directly from blood culture broth represents a simple and effective technique that can reduce the turnaround time by 24 hours, which in turn benefits the clinician in appropriate utilization of antimicrobials for better patient care. published online July 9,2021
DOI https://doi.org/

$10.1055 / \mathrm{s}-0041-1732489$ ISSN $0974-2727$
C 2021. The Indian Association of Laboratory Physicians.

This is an open access article published by Thieme under the terms of the Creative Commons Attribution-NonDerivative-NonCommercial-License, permitting copying and reproduction so long as the original work is given appropriate credit. Contents may not be used for commercial purposes, or adapted, remixed, transformed or built upon. (https://creativecommons.org/licenses/by-nc-nd/4.0/).

Thieme Medical and Scientific Publishers Pvt. Ltd. A-12, 2nd Floor, Sector 2, Noida-201301 UP, India 


\section{Introduction}

Bloodstream infections (BSIs) are medical emergencies, which can cause serious morbidities as well as mortalities. BSIs are termed as the third leading cause of health care-related infections. ${ }^{1}$ The gold standard diagnostic method for BSI is culturing the causative organism from blood and providing the antimicrobial susceptibility testing (AST) result for the same. ${ }^{2}$ This will help in timely initiation of antimicrobial therapy. But, prolonged laboratory turnaround time (TAT) can increase mortality, time of hospital stay as well as cost of therapy. In the currently used conventional methods, empirical therapy can be started based on the Gram staining done from positive blood culture bottles. ${ }^{3,4}$ And final confirmatory results are based on biochemical identification and ASTs done from colonies grown on subculture and targeted therapy is given accordingly. As the whole process takes 48 hours or even longer, the need for a robust diagnostic method arises. With every hour of delay in initiating appropriate antimicrobial agents for sepsis, there is an increase in mortality by $7.6 \%{ }^{5}$

Novel molecular methods and rapid phenotypic methods can help in faster identification, but lack the ability to identify broad range of pathogens and providing antibiotic susceptibility. Moreover, the hands-on processing time as well as monetary investments are high. So, still blood culture remains as the gold standard. Doing direct susceptibility testing (DST) on positive blood culture broth can expedite AST, at least by 24 hours. Since 1980s there have been a lot of studies aiming to standardize DST from blood culture using disc diffusion, automated and molecular testing. ${ }^{6-9}$ But the categorical error varies in different methods. DST methods are shown to be feasible from previous studies as there is good correlation with reference methods. But disc diffusion done directly from broth showed mixed results. ${ }^{9}$ With the advent of automation, laboratory work flows have been redesigned. Rapid susceptibility testing can be done from positive blood broth by automated Vitek system of Biomerieux. Automations help in standardizing the laboratory techniques with accurate results at a short TAT and also it is not labor intensive. ${ }^{10}$

Our study aims to assess the various errors associated with DST done with automated blood culture system compared with the reference method of AST that is done from bacterial colonies.

\section{Methodology}

\section{Study Isolates}

The study was conducted in a tertiary care public sector 2,200-bedded hospital in South India from March 2018 to August 2018 (6 months). All the positive aerobic blood cultures (BACT/ALERT® VIRTUO® bioMérieux, United States; aerobic) from patients suspected of having BSIs were subjected to Gram staining. Bottles showing Gram-negative organisms on Gram stain were included for analysis. Repeated isolates from the same patient's blood culture, positive blood specimens with more than one organism in Gram smear, positive blood specimens that yielded more than one isolate after subculture, positive blood specimens whose direct
Gram staining do not correlate with the growth on culture media, and positive blood specimens that yielded fastidious bacteria or yeast were excluded from the study.

\section{Procedure}

Once the blood culture bottle (BacTalert aerobic) flagged positive, it was taken out and proceeded with Gram staining. If the Gram staining was showing single morphotype of Gram-negative bacilli, the culture broth was subcultured in blood agar and MacConkey agar. After incubation for 8 hours, colonies from $5 \%$ sheep blood agar was subjected to matrix-assisted laser desorption ionization time of flight mass spectrometry (MALDI-TOF MS) for identification. Then the blood culture broth was inoculated into the Vitek-2 system. For inoculation of blood sample into the Vitek-2, the following procedures were performed.

Aspirate 8 to $15 \mathrm{~mL}$ blood sample from the blood culture bottle to $15 \mathrm{~mL}$ of gel containing tube or falcon tube. Centrifuge the tube 3,500 to 4,000 rpm for 20 minutes, using the supernatant, and make suspension in a sterile saline, McFarland range from 0.5 to 0.63 in one tube. Respective AST card was inserted based on the identification by MALDI-TOF MS (fermenter-card no. 280; nonfermenter-card no. 281) to the other tube and loaded into the Vitek-2 instrument within 20 minutes (ideally, after inoculum prepared in $\leq 30$ minutes, it has to be loaded). Result was available after 8 to 16 hours.

Antibiotics for fermenter panel included were (card no. 280) ampicillin, amoxicillin/clavulanic acid, piperacillin/ tazobactam, cefuroxime, cefuroxime axetil, ceftriaxone, cefoperazone/sulbactam, cefepime, imipenem, meropenem, amikacin, gentamicin, nalidixic acid, ciprofloxacin, tigecycline, nitrofurantoin, colistin, and trimethoprim/sulfamethoxazole.

Antibiotics for nonfermenter panel included were (card no. 281) ticarcillin/clavulanic acid, piperacillin/tazobactam, ceftazidime, cefepime, imipenem, meropenem, amikacin, gentamicin, ciprofloxacin, levofloxacin, minocycline, tigecycline, colistin, and trimethoprim/sulfamethoxazole.

Based on the reports, comment has been given by the artificial intelligence data present in the system comparing with Clinical and Laboratory Standards Institute (CLSI) and The European Committee on Antimicrobial Susceptibility Testing (EUCAST) guidelines.

Results of the DST by Vitek were compared with the AST results obtained by again doing Vitek from colonies grown on subculture plate.

The performance of DST by Vitek as compared with reference (colony) AST by Vitek was expressed in terms of categorical agreement and categorical disagreement according to ISO 20776-2:2007 guidelines. ${ }^{11}$ Whenever a test method yielded same susceptibility category as that of reference method, it is said to be categorically agreed. The categorical disagreement was further characterized into minor error $(\mathrm{mE})$, major error $(\mathrm{ME})$, and very major error (VME). When result in one method is intermediate and other method is susceptible or resistant, it is said to be $\mathrm{mE}$. When reference method yields resistant category and test method yields susceptible category, this is called as very ME. When reference method result is susceptible and test method result is 
resistant, it is said to be ME. Essential agreement for an isolate meant that minimum inhibitory concentrations (MICs) obtained for the test direct blood culture method was within \pm 1 2-fold dilution of the currently used routine method. All collected data were entered into Microsoft Excel sheet. Analysis of data was performed using SPSS software. Our study was funded by Jawaharlal Institute of Postgraduate Medical Education \& Research (JIPMER) Intramural fund.

\section{Results}

One twenty positively flagged blood cultures during the study period were included in the study. For analysis to be valid, a minimum of 30 isolates should be included. ${ }^{12}$ So, 60 isolates in Enterobacteriaceae family were included, which comprise 30 Escherichia coli and 30 Klebsiella pneumoniae. Also, 60 nonfermenters were included, with 30 Pseudomonas aeruginosa and 30 Acinetobacter baumannii.

As explained in -Table 1, nonfermenters showed a better categorical agreement of $97.6 \%$ compared with Enterobacteriaceae, which showed 97\%. Among Enterobacteriaceae, both E. coli and K. pneumoniae showed categorical agreement of $97 \%$ each. Nonfermenters behaved in a different way. P. aeruginosa showed an excellent categorical agreement of $99.4 \%$, whereas A. baumannii showed $95.8 \%$ which was least among all the four.

Assessment of categorical disagreement was also done. $\mathrm{mE}$ was highest in A. baumannii (3.8\%), ME and VME were highest in K. pneumoniae (1.2 and 0.5\%, respectively). Essential agreement was $97.1 \%$. P. aeruginosa showed an excellent essential agreement of $99.4 \%$. A. baumannii showed maximum essential disagreement (4.2\%) among the isolates tested.

For selective reporting of antibiotics, analysis of DST and AST of individual antibiotics should be done. Among Enterobacteriaceae, most of the antibiotics in the Vitek panel showed an excellent categorical agreement > 95\% except piperacillin/tazobactam and nitrofurantoin that showed only 93.3 and $85.0 \%$, respectively. Categorical disagreement was more than acceptable level (>3\%) in $\mathrm{mE}$ for amoxicillin/clavulanic acid (3.3\%), piperacillin/tazobactam (5.0\%), imipenem (3.3\%), and nitrofurantoin (13.3\%). ME and VME were showing acceptable levels of categorical disagreement (<3\%). Essential disagreement of $>3 \%$ was seen in piperacillin/tazobactam (6.7\%), imipenem (5.0\%), meropenem (3.4\%), amikacin (3.3\%), gentamicin (3.3\%), ciprofloxacin (3.4\%), and nitrofurantoin (15.0\%) as shown in - Table 2.

Nonfermenters also showed excellent categorical agreement > 95\% except levofloxacin (91.7\%) and minocycline (90.0\%). ME and VME showed excellent categorical agreement $>97 \%$, but $\mathrm{mE}$ showed categorical disagreement $>3 \%$ for ticarcillin/clavulanic acid (95.0\%), levofloxacin (8.3\%), minocycline (10.0\%), and tigecycline (3.3\%) as shown in - Table 3.

\section{Discussion}

Rapid AST for invasive infections like BSI is the need of the hour. Even though molecular mechanisms of resistance to different antibiotics can be detected by nucleic acid amplification tests, the practical utility is less. On a clinical perspective, rapid identification of antibiotics to which organism is susceptible is more important to modify the empirical antibiotics. ${ }^{13}$ Most of the studies were done based on disk diffusion method directly from blood culture broth and other clinical samples such as urine, bile, and respiratory samples. ${ }^{6,14}$ Here comes the importance of an automated AST system with software and disposable reagent cards like Vitek that has shorter TAT and gives MIC values also. There are only few studies, conducted on this idea, which implemented laborious workflow involving bacterial pellets as extra step. ${ }^{15-19}$ We have used supernatant after centrifugation as bacterial inoculums. And also most of the similar studies have used AST-N020, AST-202, AST-NO09 Vitek cards. We have used card no. N280 for fermenter organism and card no. N281 for nonfermenter

Table 1 Performance of direct DST test compared with reference (colony) AST method test by automated VITEK-2 System

\begin{tabular}{|c|c|c|c|c|c|c|c|}
\hline \multirow{2}{*}{$\begin{array}{l}\text { Organisms and antibiotic } \\
\text { tested } \\
\left(n^{*} A b=N\right)\end{array}$} & \multirow{2}{*}{$\begin{array}{l}\text { Categorical } \\
\text { agreement, } \\
n(\%)\end{array}$} & \multicolumn{4}{|c|}{$\begin{array}{l}\text { Categorical disagreement, } n(\%) \text { among } \\
\text { isolate-antibiotic combinations tested }\end{array}$} & \multicolumn{2}{|c|}{ Essential agreement } \\
\hline & & Minor & Major & $\begin{array}{l}\text { Very } \\
\text { major }\end{array}$ & Total & Agreed & Disagreed \\
\hline $\begin{array}{l}\text { Enterobacteriaceae } \\
\left(60^{*} 18=1,080\right)\end{array}$ & $1048(97.0 \%)$ & $16(1.4 \%)$ & $12(1.1 \%)$ & $4(0.4 \%)$ & $32(3.0 \%)$ & $1,044(97.7 \%)$ & $36(3.3 \%)$ \\
\hline $\begin{array}{l}\text { Escherichia coli } \\
\left(30^{*} 18=540\right)\end{array}$ & $524(97.0 \%)$ & $10(1.8 \%)$ & $5(0.9 \%)$ & $1(0.1 \%)$ & $16(3.0 \%)$ & $521(96.4 \%)$ & $19(3.6 \%)$ \\
\hline $\begin{array}{l}\text { Klebsiella pneumoniae } \\
\left(30^{*} 18=540\right)\end{array}$ & $524(97.0 \%)$ & $6(1.1 \%)$ & $7(1.2 \%)$ & $3(0.5 \%)$ & $16(3.0 \%)$ & $523(96.7 \%)$ & $17(3.3 \%)$ \\
\hline $\begin{array}{l}\text { Nonfermenters } \\
\left(60^{*} 15=900\right)\end{array}$ & $878(97.6 \%)$ & $19(2.1 \%)$ & $2(0.2 \%)$ & $1(0.1 \%)$ & $22(2.4 \%)$ & $878(97.6 \%)$ & $22(2.4 \%)$ \\
\hline $\begin{array}{l}\text { Pseudomonas aeruginosa } \\
\left(30^{*} 15=450\right)\end{array}$ & 447 (99.4\%) & $2(0.4 \%)$ & $1(0.2 \%)$ & $0(0.0)$ & $3(0.6 \%)$ & 447 (99.4\%) & $3(0.6 \%)$ \\
\hline $\begin{array}{l}\text { Acinetobacter baumannii } \\
\left(30^{*} 15=450\right)\end{array}$ & 431 (95.8\%) & $17(3.8 \%)$ & $1(0.2 \%)$ & $1(0.2 \%)$ & $19(4.2 \%)$ & 431 (95.8\%) & $19(4.2 \%)$ \\
\hline
\end{tabular}

Abbreviations: AST, antimicrobial susceptibility testing; DST, direct susceptibility testing. 
Direct Susceptibility Testing by VITEK-2 Kavipriya et al. 377

Table 2 Performance of direct DST test compared with reference (colony) AST method test for Enterobacteriaceae by VITEK-2 system

\begin{tabular}{|c|c|c|c|c|c|c|c|}
\hline \multirow{2}{*}{$\begin{array}{l}\text { Enterobacteriaceae } \\
(60)\end{array}$} & \multirow{2}{*}{$\begin{array}{l}\text { Categorical } \\
\text { agreement, } \\
n(\%)\end{array}$} & \multicolumn{4}{|c|}{ Categorical disagreement $n(\%)$} & \multicolumn{2}{|c|}{ Essential agreement } \\
\hline & & Minor & Major & $\begin{array}{l}\text { Very } \\
\text { major }\end{array}$ & Total & Agreed & Disagreed \\
\hline Ampicillin & $60(100.0 \%$ & $0(0.0 \%)$ & $0(0.0 \%)$ & $0(0.0 \%)$ & $0(0.0 \%)$ & $60(100.0 \%)$ & $0(0.0 \%)$ \\
\hline $\begin{array}{l}\text { Amoxicillin/ clavulanic } \\
\text { acid }\end{array}$ & $58(96.7 \%)$ & $2(3.3 \%)$ & $0(0.0 \%)$ & $0(0.0 \%)$ & $2(3.3 \%)$ & $58(96.7 \%)$ & $2(3.3 \%)$ \\
\hline $\begin{array}{l}\text { Piperacillin/ } \\
\text { tazobactam }\end{array}$ & $56(93.3 \%)$ & $3(5.0 \%)$ & $1(1.7 \%)$ & $0(0.0 \%)$ & $4(6.7 \%)$ & $56(93.3 \%)$ & $4(6.7 \%)$ \\
\hline Cefuroxime & $59(98.3 \%)$ & $0(0.0)$ & $1(1.7 \%)$ & $0(0.0 \%)$ & $1(1.7 \%)$ & 59 (98.3\%) & $1(1.7 \%)$ \\
\hline Cefuroxime axetil & $59(98.3 \%)$ & $0(0.0)$ & $1(1.7 \%)$ & $0(0.0)$ & $1(1.7 \%)$ & $59(98.3 \%)$ & $1(1.7 \%)$ \\
\hline Ceftriaxone & $59(98.3 \%)$ & $0(0.0)$ & $1(1.7 \%)$ & $0(0.0)$ & $1(1.7 \%)$ & $59(98.3 \%)$ & $1(1.7 \%)$ \\
\hline $\begin{array}{l}\text { Cefoperazone/ } \\
\text { sulbactam }\end{array}$ & $59(98.3 \%)$ & $0(0.0)$ & $1(1.7 \%)$ & $0(0.0)$ & $1(1.7 \%)$ & $59(98.3 \%)$ & $1(1.7 \%)$ \\
\hline Cefepime & $59(98.3 \%)$ & $0(0.0)$ & $0(0.0)$ & $1(1.7 \%)$ & $1(1.7 \%)$ & 59 (98.3\%) & $1(1.7 \%)$ \\
\hline Imipenem & 57 (95.0\%) & $2(3.3 \%)$ & $1(1.7 \%)$ & $0(0.0 \%)$ & $3(5.0 \%)$ & 57 (95.0\%) & $3(5.0 \%)$ \\
\hline Meropenem & $58(96.6 \%)$ & $0(0.0)$ & $1(1.7 \%)$ & $1(1.7 \%)$ & $2(3.4 \%)$ & 58 (96.6\%) & $2(3.4 \%)$ \\
\hline Amikacin & $59(98.3 \%)$ & $0(0.0)$ & $1(1.7 \%)$ & $0(0.0)$ & $1(1.7 \%)$ & 58 (96.7\%) & $2(3.3 \%)$ \\
\hline Gentamicin & $59(98.3 \%)$ & $0(0.0)$ & $1(1.7 \%)$ & $0(0.0)$ & 1 (1.7\%) & 58 (96.7\%) & $2(3.3 \%)$ \\
\hline Nalidixic acid & $60(100.0 \%)$ & $0(0.0)$ & $0(0.0)$ & $0(0.0)$ & $0(0.0)$ & $60(100.0 \%)$ & $0(0.0)$ \\
\hline Ciprofloxacin & 58 (96.6\%) & $1(1.7 \%)$ & $1(1.7 \%)$ & $0(0.0)$ & $2(3.4 \%)$ & 58 (96.6\%) & $2(3.4 \%)$ \\
\hline Tigecycline & 59 (98.3\%) & $0(0.0)$ & $1(1.7 \%)$ & $0(0.0)$ & $1(1.7 \%)$ & 59 (98.3\%) & $1(1.7 \%)$ \\
\hline Nitrofurantoin & $51(85.0 \%)$ & $8(13.3)$ & $0(0.0)$ & $1(1.6 \%)$ & $9(15.0 \%)$ & $51(85.0 \%)$ & $9(15.0 \%)$ \\
\hline Colistin & 59 (98.3\%) & $0(0.0)$ & $1(1.7 \%)$ & $0(0.0)$ & $1(1.7 \%)$ & 59 (98.3\%) & $1(1.7 \%)$ \\
\hline $\begin{array}{l}\text { Trimethoprim/ } \\
\text { sulfamethoxazole }\end{array}$ & 59 (98.3\%) & $0(0.0)$ & $0(0.0)$ & $1(1.7 \%)$ & $1(1.7 \%)$ & 59 (98.3\%) & $1(1.7 \%)$ \\
\hline
\end{tabular}

Abbreviations: AST, antimicrobial susceptibility testing; DST, direct susceptibility testing.

organism that were easily available and routinely used card in most of the laboratories.

Barnini et $\mathrm{al}^{15}$ conducted similar study in Italy in 2014, where they used MALDI-TOF MS for identification and AST by Vitek-2. But their accordance of direct AST result with culture AST was less than our study. Usage of serum separator tubes or short-term subculture in liquid medium may be the reason for that. In Belgium, Maelegheer and Nulens ${ }^{16}$ did similar study in 2016, where they got a better concordance of $98.9 \%$ than our study. They microcentrifuged positive blood sample and MALDI-TOF identification and AST by BD Phoenix was performed. Study by Mauri et $\mathrm{al}^{17}$ showed an excellent categorical agreement of $98.1 \%$ and essential agreement of $97.7 \%$. They used MALDI-TOF mass spectrometry identification and automated AST.

Among the isolates tested, $A$. baumannii showed maximum essential disagreement (4.2\%). mE was highest in A. baumannii, whereas ME and VME were highest in K. pneumoniae, but they were within the acceptable range $(<3 \%)$. But in study by Mauri et al, ME and VME were not reported in K. pneumoniae. Analysis of drug-bug combination showed essential agreement less than allowed level (<95\%) in Enterobacteriaceaepiperacillin, tazobactam, and nitrofurantoin, with no ME and
VME. Amoxiclav, Piptaz, and nitrofurantoin were showing $\mathrm{mE}$ for Enterobacteriaceae, which was observed in study done by Mauri et al also. ${ }^{17}$ In nonfermenters, levofloxacin and minocycline showed essential agreement less than 95\%. MEs and VMEs were not reported. Pan et al showed less essential agreement (<95\%) for meropenem, imipenem, cefepime, ceftriaxone, and ceftazidime, which was not reflected in our study. ${ }^{18}$

In this study we demonstrated that Vitek-2 can be used to provide rapid, highly accurate susceptibility reports directly from positive blood cultures with Enterobacteriaceae and nonfermenters. The advantages of using Vitek include widespread availability in majority of laboratories around the globe, so that the need for additional equipment and facilities can be circumvented. Less hands-on time and low additional cost are the added advantages. Moreover, there is no need of dedicated manpower to perform the test. ${ }^{19}$ In a study conducted by Höring et al, comparing Vitek-2 and BD Phoenix for direct blood culture inoculation, Vitek-2 demonstrated higher test accuracy. ${ }^{20}$

Finally, in addition to the high performance for rapid AST, our method reduced the TAT also considerably. 
Table 3 Performance of direct test compared with reference (colony) test for nonfermenters by VITEK-2 system

\begin{tabular}{|l|l|l|l|l|l|l|l|}
\hline \multirow{2}{*}{$\begin{array}{l}\text { Nonfermenter } \\
(60)\end{array}$} & \multirow{2}{*}{$\begin{array}{l}\text { Categorical } \\
\text { agreement (\%) }\end{array}$} & \multicolumn{3}{|c|}{ Categorical disagreement (\%) } & \multicolumn{2}{c|}{ Essential agreement } \\
\cline { 3 - 7 } & Minor & Major & Very major & Total & Agreed & Disagreed \\
\hline Ticarcillin/clavulanic acid & $57(95.0 \%)$ & $3(5.0 \%)$ & $0(0.0)$ & $0(0.0)$ & $3(5.0 \%)$ & $57(95.0 \%)$ & $3(5.0 \%)$ \\
\hline Piperacillin/tazobactam & $59(98.3 \%)$ & $0(0.0)$ & $1(1.7 \%)$ & $0(0.0)$ & $1(1.7 \%)$ & $59(98.3 \%)$ & $1(1.7 \%)$ \\
\hline Ceftazidime & $60(100.0 \%)$ & $0(0.0)$ & $0(0.0)$ & $0(0.0)$ & $0(0.0)$ & $60(100.0 \%)$ & $0(0.0)$ \\
\hline Cefoperazone/sulbactam & $59(98.3 \%)$ & $1(1.7 \%)$ & $0(0.0)$ & $0(0.0)$ & $1(1.7 \%)$ & $59(98.3 \%)$ & $1(1.7 \%)$ \\
\hline Cefepime & $60(100.0 \%)$ & $0(0.0)$ & $0(0.0)$ & $0(0.0)$ & $0(0.0)$ & $60(100.0 \%)$ & $0(0.0)$ \\
\hline Doripenem & $60(100.0 \%)$ & $0(0.0)$ & $0(0.0)$ & $0(0.0)$ & $0(0.0)$ & $60(100.0 \%)$ & $0(0.0)$ \\
\hline Imipenem & $60(100.0 \%)$ & $0(0.0)$ & $0(0.0)$ & $0(0.0)$ & $0(0.0)$ & $60(100.0 \%)$ & $0(0.0)$ \\
\hline Meropenem & $60(100.0 \%)$ & $0(0.0)$ & $0(0.0)$ & $0(0.0)$ & $0(0.0)$ & $60(100.0 \%)$ & $0(0.0)$ \\
\hline Amikacin & $59(98.3 \%)$ & $0(0.0)$ & $0(0.0)$ & $1(1.7 \%)$ & $1(1.7 \%)$ & $59(98.3 \%)$ & $1(1.7 \%)$ \\
\hline Gentamicin & $59(98.3 \%)$ & $1(1.7 \%)$ & $0(0.0)$ & $0(0.0)$ & $1(1.7 \%)$ & $59(98.3 \%)$ & $1(1.7 \%)$ \\
\hline Ciprofloxacin & $59(98.3 \%)$ & $1(1.7 \%)$ & $0(0.0)$ & $0(0.0)$ & $1(1.7 \%)$ & $59(98.3 \%)$ & $1(1.7 \%)$ \\
\hline Levofloxacin & $55(91.7 \%)$ & $5(8.3 \%)$ & $0(0.0)$ & $0(0.0)$ & $5(8.3 \%)$ & $55(91.7 \%)$ & $5(8.3 \%)$ \\
\hline Minocycline & $54(90.0 \%)$ & $6(10.0 \%)$ & $0(0.0)$ & $0(0.0)$ & $6(10.0 \%)$ & $54(90.0 \%)$ & $6(10.0 \%)$ \\
\hline Tigecycline & $57(95.0 \%)$ & $2(3.3 \%)$ & $1(1.6 \%)$ & $0(0.0)$ & $3(5.0 \%)$ & $57(95.0 \%)$ & $3(5.0 \%)$ \\
\hline Colistin & $60(100.0 \%)$ & $0(0.0)$ & $0(0.0)$ & $0(0.0)$ & $0(0.0)$ & $60(100.0 \%)$ & $0(0.0)$ \\
\hline
\end{tabular}

\section{Conclusion}

The new procedure of doing direct AST from blood culture represents a simple, effective technique that can reduce the TAT by 24 hours, which in turn benefits the patient outcome by reducing the mortality, time to de-escalation, length of stay, and antibiotic-related side effects like Clostridium difficile infection.

\section{Conflict of Interest}

None.

\section{References}

1 Lemos TC, Cogo LL, Maestri AC, Hadad M, Nogueira KDS. Is it possible to perform bacterial identification and antimicrobial susceptibility testing with a positive blood culture bottle for quick diagnosis of bloodstream infections? Rev Soc Bras Med Trop 2018;51(2):215-218

2 Klouche M, Schröder U. Rapid methods for diagnosis of bloodstream infections. Clin Chem Lab Med 2008;46(7):888-908

3 Søgaard M, Nørgaard M, Schønheyder HC. First notification of positive blood cultures and the high accuracy of the gram stain report. J Clin Microbiol 2007;45(4):1113-1117

4 Nain J, Deepashree R, Tamang P, et al. Comparison of four different methods of smear preparation for gram staining of positively flagged automated blood culture bottles. J Curr Res Sci Med 2018;4(2):98

5 Kumar A, Roberts D, Wood KE, et al. Duration of hypotension before initiation of effective antimicrobial therapy is the critical determinant of survival in human septic shock. Crit Care Med 2006;34(6):1589-1596

6 Rajshekar D, Chaudhari KV, Bhat P, et al. Evaluation of performance of direct disk diffusion test from positively flagged blood culture broth: a large scale study from South India. J Lab Physicians 2019;11(2):154-160

7 Hazelton B, Thomas LC, Olma T, et al. Rapid and accurate direct antibiotic susceptibility testing of blood culture broths using MALDI Sepsityper combined with the BD Phoenix automated system. J Med Microbiol 2014;63(Pt 12):1590-1594
8 Heather CS, Maley M. Automated direct screening for resistance of Gram-negative blood cultures using the BD Kiestra WorkCell. Eur J Clin Microbiol Infect Dis 2018;37(1):117-125

9 Coyle MB, McGonagle LA, Plorde JJ, Clausen CR, Schoenknecht FD. Rapid antimicrobial susceptibility testing of isolates from blood cultures by direct inoculation and early reading of disk diffusion tests. J Clin Microbiol 1984;20(3):473-477

10 Jorgensen JH. Selection criteria for an antimicrobial susceptibility testing system. J Clin Microbiol 1993;31(11):2841-2844

11 std . ISO 20776-1:2006-Clinical laboratory testing and in vitro diagnostic test systems-Susceptibility testing of infectious agents and evaluation of performance of antimicrobial susceptibility test devices-part 2: Evaluation of performance of antimicrobial susceptibility test devices. Available at: https://www.iso.org/standard/41631.html. Accessed June 17, 2021/std

12 Jenkins SG, Schuetz AN. Current concepts in laboratory testing to guide antimicrobial therapy. Mayo Clin Proc 2012;87(3):290-308

13 Hogan CA, Watz N, Budvytiene I, Banaei N. Rapid antimicrobial susceptibility testing by VITEK®2 directly from blood cultures in patients with Gram-negative rod bacteremia. Diagn Microbiol Infect Dis 2019;94(2):116-121

14 Coorevits L, Boelens J, Claeys G. Direct susceptibility testing by disk diffusion on clinical samples: a rapid and accurate tool for antibiotic stewardship. Eur J Clin Microbiol Infect Dis 2015;34(6):1207-1212

15 Barnini S, Brucculeri V, Morici P, Ghelardi E, Florio W, Lupetti A. A new rapid method for direct antimicrobial susceptibility testing of bacteria from positive blood cultures. BMC Microbiol 2016;16(1):185

16 Maelegheer K, Nulens E. Same-day identification and antibiotic susceptibility testing on positive blood cultures: a simple and inexpensive procedure. Eur J Clin Microbiol Infect Dis 2017;36(4):681-687

17 Mauri C, Principe L, Bracco S, et al. Identification by mass spectrometry and automated susceptibility testing from positive bottles: a simple, rapid, and standardized approach to reduce the turnaround time in the management of blood cultures. BMC Infect Dis 2017;17(1):749 
18 Pan H, Li W, Li R, Li Y, Zhang Y, Sun E. Simple sample preparation method for direct microbial identification and susceptibility testing from positive blood cultures. Front Microbiol 2019;0510.3389/fmicb.2018.00481

19 Machen A, Drake T, Wang YF. Same day identification and full panel antimicrobial susceptibility testing of bacteria from positive blood culture bottles made possible by a combined lysis-filtration method with MALDI-TOF VITEK mass spectrometry and the VITEK2 system. PLoS One 2014;9(2):e87870

20 Höring S, Massarani AS, Löffler B, Rödel J. Rapid antibiotic susceptibility testing in blood culture diagnostics performed by direct inoculation using the VITEK ${ }^{\circledR}-2$ and BD Phoenix ${ }^{\mathrm{TM}}$ platforms. Eur J Clin Microbiol Infect Dis 2019;38(3):471-478 